\title{
Contribution à l'étude des procédés de fabrication et des caractéristiques physico-chimiques et sensorielles du fromage Mashanza produit au Sud- Kivu
}

\author{
Birali Mwamini1, 2, Sumbu Zola², Walangululu Jean'1 \\ 1 Université Catholique de Bukavu (UCB), Faculté des Sciences agronomiques, BP. 02, Cyangugu, Rwanda, \\ 2Université de Kinshasa (UNIKIN), Faculté d'Agronomie, B.P. 117, Kinshasa I, RD. Congo. \\ Corresponding Author: Birali, M.J.; birali.jennifer@gmail.com; +243994646409
}

Original submitted in on $9^{\text {th }}$ September 2019. Published online at www.m.elewa.org/journals/ on 31st December 2019 https://doi.org/10.35759/JABs.v144.6

\section{RESUME}

Objectif : Mashanza est un fromage frais traditionnel au lait de vache, fabriqué artisanalement au Sud-Kivu et dans d'autres provinces, mais dont le développement fait face à une connaissance scientifique insuffisante des procédés et de la qualité et à l'absence de norme. Ainsi, l'objectif de cette étude est d'analyser les procédés de fabrication et d'évaluer les paramètres de qualité et le rendement du Mashanza. Méthodologie et Résultats: Un suivi de la fabrication a été réalisé in situ et des analyses physicochimiques et sensorielles ont été effectuées sur le produit après fabrication dans 12 unités de fabrication à Kabare (Ucb, Comis, Miti), Kalehe (Luk, Luz, Mag), Uvira (Kond, Shuk, Zia) et Walungu (Cab, Cing, Uprelo). Le pH et la température au cours de la fermentation ont été déterminés. Les procédés utilisés par les 12 unités de fabrication varient en fonction de la source d'approvisionnement du lait, du traitement thermique, de la quantité de lactosérum utilisée comme ferment, de la durée de fermentation, des équipements utilisés ainsi que du mode d'égouttage. Les valeurs moyennes obtenues sont dans la gamme de 31 à $48,8 \%$ de matière sèche, 20 à $43 \%$ de protéines, 2,5 à 3,9\% des cendres totales, et de 4,9 à 6,2 $\mathrm{mg} / \mathrm{g}$ de matière sèche pour le phosphore, de 0,8 à 3,3 pour le Sodium et de 5,7 à 17,5 pour le Potassium. Le $\mathrm{pH}$ varie de 3,5 à 3,8 , l'activité de l'eau (Aw) de 0,79 à 0,95 et enfin, l'acidité lactique, de $41,5^{\circ} \mathrm{D}$ à $100,8^{\circ} \mathrm{D}$. Concernant l'évaluation sensorielle des fromages, en général, tous ont été bien appréciés du point de vue saveur, texture et couleur.

Conclusion et application des résultats : Les procédés utilisés dans les 12 unités de fabrication varient en fonction de la source d'approvisionnement du lait, du traitement thermique, de la quantité de lactosérum utilisée comme ferment, de la durée de fermentation, des équipements utilisés ainsi que du mode d'égouttage. Les résultats obtenus permettront de servir de base pour la recherche des normes du Mashanza et de compléter les connaissances sur les procédés et les aspects qualitatifs. Par ailleurs, ils ouvrent la voie sur le choix des procédés pouvant s'adapter au niveau d'investissement des producteurs, tout en prenant en compte les facteurs pouvant influencer la qualité.

Mots-clés : fromage frais, Mashanza, acidification, qualité, rendement. 


\title{
Contribution to the study of manufacturing processes and physicochemical and sensory characteristics of Mashanza cheese produced in South Kivu
}

\begin{abstract}
Objective: The objective of this study is to analyse the manufacturing processes and evaluate the quality parameters and performance of the Mashanza for possible standardization.

Methodology and Results: To do this, a follow-up of the transformation process was carried out in situ while physicochemical and sensory analyses were carried out on the product after manufacturing in 12 units in Kabare (Ucb, Comis, Miti), Kalehe (Luk, Luz, Mag), Uvira (Kond, Shuk, Zia) and Walungu (Cab, Cing, Uprelo). Acidification kinetics were determined for $\mathrm{pH}$ and temperature. The average values found are in the range of 31 to $48.8 \%$ dry matter, the proteins from 20 to $43 \%$, the total ash, from 2.5 to $3.9 \%$ with values from 4.9 to $6,2 \mathrm{mg} / \mathrm{g}$ dry matter for phosphorus, 0.8 to 3.3 for Sodium and 5.7 to 17.5 for Potassium. The $\mathrm{pH}$ varies from 3.5 to 3.8 , the water activity Aw from 0.79 to 0.95 and finally, the lactic acidity, from $41.5^{\circ}$ to $100.8^{\circ}$. Regarding the sensory evaluation of cheeses, in general, all were well appreciated from the point of view of flavour, texture and colour.

Conclusions and application of findings: Processes used by the 12 manufacturing units vary according to the milk supply source, the heat treatment, the amount of whey used as ferment, the fermentation time, the equipment used and the dewatering mode. The results obtained will serve as a basis for the search for "Mashanza" standards and to complete the knowledge of the processes and the qualitative aspects. Moreover, they open the way to the choice of processes that can adapt to the level of investment of producers, taking into account the factors influencing the quality of the Mashanza.
\end{abstract}

Keywords: fresh cheese, Mashanza, acidification, quality, yield.

\section{INTRODUCTION}

Considéré comme une denrée sensible, le lait revêt en Afrique un caractère hautement stratégique (Diop, 1996). Par sa richesse en protéines de bonne qualité, en matière grasse, en calcium et en vitamines, le fromage représente un aliment de haute qualité nutritionnelle (Vivegnis et al., 1998; Roudaut et Lefrancq, 2005). Les bactéries lactiques jouent un rôle déterminant dans l'acidification du lait et la fabrication des fromages, par le renforcement de la vitesse de coagulation et l'augmentation de la synérèse. La croissance et la succession des bactéries, étant sous l'influence de la température (Jamet, 2009). Les technologies fromagères peuvent différer en fonction des cinétiques d'égouttage et d'acidification (Jeantet et al., 2008). Le Mashanza est un fromage frais produit artisanalement au Sud-Kivu et dans d'autres provinces voisines (Nord-Kivu, Ituri), à l'est de la RDC. II est

\section{MATERIELS ET METHODES}

Milieu d'étude : L'étude a été réalisée de septembre à novembre 2018 dans la province du Sud-Kivu à l'est de la RD Congo, située entre $1^{\circ} 36^{\prime}$ et $5^{\circ}$ de latitude Sud et $26^{\circ} 47^{\prime}$ et $29^{\circ} 20^{\prime}$ de longitude Est. La température moyenne annuelle est de $19^{\circ} \mathrm{C}$, et l'altitude varie entre $773 \mathrm{~m}$ et $3.000 \mathrm{~m}$. L'étude s'est intéressée à 4 consommé seul ou accompagné, le plus souvent de féculents comme le foufou ou la patate douce. Cependant son industrialisation et sa commercialisation se heurtent à divers obstacles, notamment une connaissance scientifique insuffisante des procédés et de la qualité, l'absence de norme, une durée de vie brève en conditions ambiantes. La maîtrise des étapes d'acidification et d'égouttage du Mashanza pendant la fabrication permettrait d'assurer une qualité régulière et d'orienter les caractéristiques globales du produit. Ainsi, l'objectif de la présente étude est d'analyser les procédés de fabrication dans 12 fermes et d'évaluer les paramètres de qualité et le rendement du Mashanza. Et spécifiquement: (i) comparer les procédés de fabrication du Mashanza, (ii) évaluer la qualité du produit sur le plan physico-chimique et sensoriel, (iii) et enfin établir le rendement technologique.

territoires (Kabare, Kalehe, Uvira et Walungu). La plaine de la Ruzizi, dans le territoire d'Uvira, connaît un climat tropical à tendance sèche, autour de $1.000 \mathrm{~mm}$ par an (Ministère du Plan, 2005). Le tableau 1 donne les coordonnées géographiques des 12 unités de fabrication. 
Tableau 1 : Coordonnées géographiques des 12 fermes

\begin{tabular}{|c|c|c|}
\hline Nom & Territoire & Coordonnées géographiques \\
\hline UCB & Kabare & Altitude : $1701 \mathrm{~m} ;$ Latitude : $02.28977^{\circ} ;$ Longitude: $028.82176^{\circ}$ \\
\hline Comis & Kabare & Altitude : $1706 \mathrm{~m} ;$ Latitude:02.28716º ; Longitude:028.82007 \\
\hline Miti & Kabare & Altitude : $1584 \mathrm{~m} ;{\text { Latitude: } 02.36128^{\circ} ; \text { Longitude:028.79142 }}^{\circ}$ \\
\hline Kond & Uvira & Altitude : $945 \mathrm{~m}$; Latitude:02.90645 ; Longitude:029.05540 \\
\hline Zia & Uvira & 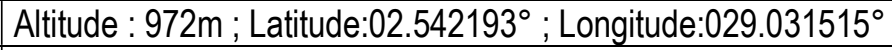 \\
\hline Shuk & Uvira & Altitude : $945 \mathrm{~m} ;$ Latitude: $02.542316^{\circ} ;$ Longitude: $029.031385^{\circ}$ \\
\hline Luk & Kalehe & Altitude : $1480 \mathrm{~m} ;$ Latitude : $02.653^{\circ}$; Longitude : $028.91905^{\circ}$ \\
\hline Mag & Kalehe & Altitude : $1478 \mathrm{~m} ;$ Latitude : 02.11727 ; Longitude : $028.89541^{\circ}$ \\
\hline Luz & Kalehe & Altitude: $1507 \mathrm{~m} ;$; Latitude:02.122265 ; Longitude:029.87343 \\
\hline Cing & Walungu & Altitude : $2400 \mathrm{~m}$; Latitude:02.49273 $;$ Longitude:028.69902 ${ }^{\circ}$ \\
\hline Cab & Walungu & Altitude : $2474 \mathrm{~m} ;$ Latitude:02.51944º ; Longitude:028.68505 \\
\hline Uprelo & Walungu & Altitude : $2521 \mathrm{~m} ;$ Latitude: $02.48305^{\circ}$; Longitude:028.67459 \\
\hline
\end{tabular}

Matériels : Pour le suivi de la fabrication de Mashanza, les échantillons de Mashanza, le lactosérum employé comme ferment et le lait ont été récoltés et analysés.
Avant la fabrication, les valeurs de $\mathrm{pH}$ et de températures du lait et du lactosérum ont été déterminées, elles sont reprises dans le tableau 2.

Tableau 2: Valeurs de pH et températures moyennes du lait frais et de lactosérum de 12 fermes.

\begin{tabular}{|c|c|c|c|c|c|c|c|c|c|c|c|c|c|}
\hline \multirow{3}{*}{\multicolumn{2}{|c|}{ MATERIELS }} & \multicolumn{12}{|c|}{ Localisation } \\
\hline & & \multicolumn{3}{|c|}{ Kabare } & \multicolumn{3}{|c|}{ Kalehe } & \multicolumn{3}{|c|}{ Uvira } & \multicolumn{3}{|c|}{ Walungu } \\
\hline & & Comis & Miti & UCB & Mag & Luk & Luz & Shuk & Kond & Zia & Cing & Uprelo & Cab \\
\hline \multirow[t]{2}{*}{ Lait } & $\mathrm{pH}$ & 6,5 & 6,3 & 6,5 & 6,5 & 6,7 & 6,8 & 6,3 & 6,8 & 6,7 & 6,3 & 6,2 & 6,8 \\
\hline & $\mathrm{T}^{\circ}$ & 32,5 & 31,7 & 32,2 & 27,7 & 25,8 & 30,6 & 31 & 31,7 & 30,4 & 26 & 32,8 & 37,1 \\
\hline \multirow[t]{2}{*}{ Ferment } & $\mathrm{pH}$ & 3,5 & 3,6 & 3,5 & 3,9 & 4,1 & 4 & 3,6 & 3,9 & 3,9 & 3,5 & 3,6 & 4,03 \\
\hline & $T^{\circ}$ & 21,2 & 21,2 & 18,6 & 19,9 & 20,1 & 17,4 & 25,4 & 27,1 & 28,9 & 16,3 & 17,8 & 14,1 \\
\hline
\end{tabular}

Méthodes: Une enquête a été réalisée dans 4 territoires (Kabare, Kalehe, Uvira et Walungu) où la fabrication du Mashanza est ancrée dans la tradition des communautés. Au total 12 producteurs dont 9 fermiers et 3 artisans ont été ciblés dont 4 par territoire, à la suite d'une étude antérieure. Leur production a été suivie pendant une fermentation de 24 heures et les paramètres de suivi ont été prélevés. Les principaux critères de choix des producteurs ont été les suivants : (i) ancienneté dans la production du Mashanza, (ii) pérennité et viabilité de l'activité toute l'année, (iii) capacité de traitement d'au moins 20 litres par opération ou par jour, (iv) accessibilité par véhicule, (v) site non éloigné de la route principale, (vi) distance de moins de 30 minutes en véhicule, d'une unité de fabrication à une autre dans le même territoire afin de permettre d'effectuer le suivi de la fabrication du Mashanza durant la même période.

NB. Certains fabricants ont préféré que leurs unités de fabrication restent dans l'anonymat.

Suivi du processus de fabrication: En vue de collecter les informations relatives à la fabrication du fromage Mashanza, une fiche d'entretien a été conçue et complétée pendant le suivi du processus qui a duré environ 24 heures dans chaque territoire pour les 3 unités retenues. Les informations étaient en rapport avec toutes les étapes de fabrication. Durant le processus, des prélèvements de température et d'humidité relative de l'ambiance ainsi que de la température et du $\mathrm{pH}$ dans la cuve de fermentation ont été réalisés au temps initial et après 2, 4, 6, 8, 22 et 24 heures.

$\mathrm{pH}$ : Le pH est mesuré à l'aide d'un pH-mètre de marque " ATC ». Avant chaque mesure, l'électrode du pH-mètre est nettoyée avec de l'eau distillée et séchée avec une serviette en papier. Un contrôle sur la fiabilité du pH-mètre est effectué avant chaque mesure, par étalonnage de l'appareil à l'aide d'une solution tampon 
de $\mathrm{pH}$ connu (7,00). Ensuite la mesure est faite par immersion du bout de l'électrode dans le lait. La valeur du pH s'affiche immédiatement sur l'écran.

Température: La détermination de la température dans la cuve de fermentation a été réalisée grâce au thermomètre à laser de marque Stil $\left(-38^{\circ} \mathrm{C}\right.$ à $\left.+365^{\circ} \mathrm{C}\right)$ et consistait à tenir le mesureur par son manche et maintenir le capteur d'infrarouges vers l'objet dont l'on souhaite mesurer la température, ensuite appuyer et soutenir la gâchette pendant environ 8 secondes pour avoir une mesure fixe sur l'écran.

Hygrométrie et température de la chambre de fermentation: Un hygro-thermomètre de marque «Brannan» a été utilisé pour recueillir la température et I'humidité relative de l'ambiance dans les salles de fermentation où se déroulait la fermentation $\mathrm{du}$ Mashanza.

Détermination des paramètres physico-chimiques de lactosérum et de Mashanza: Les analyses physico-chimiques ont été effectuées aux Laboratoires de l'Office Congolais de Contrôle (OCC), des Sciences du sol à l'Université Catholique de Bukavu (UCB) et de I'International Institute for Tropical Agriculture (IITA). Les prélèvements ont été collectés dans les bouteilles en plastiques stériles pour le lactosérum et dans les sachets stériles pour le Mashanza, puis acheminés immédiatement dans une glacière vers le laboratoire pour être réfrigérés avant analyse. Les paramètres suivants ont été concernés: $\mathrm{pH}$, matière sèche, activité de l'eau (Aw), acidité lactique, protéine, cendres, sodium, phosphore. Les paramètres suivants ont été concernés: $\mathrm{pH}$, matière sèche, activité de l'eau (Aw), acidité lactique, protéine, cendres, sodium, phosphore. Le $\mathrm{pH}$ a été mesuré à l'aide d'un $\mathrm{pH}$-mètre électronique muni d'une électrode combinée et préalablement étalonnée. La méthode consistait à plonger l'électrode dans la solution de l'échantillon de lactosérum ou dans la solution homogénéisée de $5 \mathrm{~g}$ de Mashanza avec de l'eau distillée et à lire la valeur de pH sur l'écran du pHmètre. Le taux de matière sèche a été obtenu après séchage des échantillons à l'étuve à $103^{\circ} \mathrm{C}$ pendant 24 heures, jusqu'à poids constat, et pesée des échantillons frais et ceux séchées à l'étuve et calculé suivant la formule:

$\% \mathrm{MS}=\frac{P 3-P 1}{P 2-P 1} \times 100$; où $\mathrm{P} 1$ est le poids de la

capsule vide, P2 est le poids de la capsule et de l'échantillon avant étuvage, et P3 est le poids de la capsule et de l'échantillon après dessiccation.

La teneur en cendre a été obtenue par incinération de l'échantillon par voie sèche. Deux grammes de l'échantillon placé dans un creuset en porcelaine préalablement taré étaient calcinés dans un four à moufle à $550^{\circ} \mathrm{C}$. La teneur en cendre a été trouvée par la formule suivante :

$\%$ Cendre totale $=\left(\frac{M C}{M h}\right) * 100$. Avec $: M c=$ masse de la cendre (podis après calcination-poids du creuset) et $\mathrm{Mh}=$ masse de l'échantillon humide (poids de prise contenue dans le creuset - poids du creuset)

L'Activité de l'eau a été déterminée à l'aide de l'appareil LabStart-aw de marque Novasina. Pour chaque mesure, l'échantillon est placé dans la coupelle et déposé dans la chambre de mesure en prenant soin de refermer le capot. Ensuite le bouton de démarrage est enclenché et la valeur est affichée sur l'écran.

L'acidité lactique est obtenue par titrimétrie avec de l'hydroxyde de sodium en utilisant le phénolphtaléine comme indicateur coloré, à raison de $1 \mathrm{ml}$. L'acidité Dornic correspondant à $0,1 \mathrm{~g} / \mathrm{L}$ (Goudédranche, et al., 2001). La teneur en acidité lactique s'exprime en pour cent du poids, avec 2 décimales, et se calcule comme suit :

$\%$ acide lactique $=\frac{\mathrm{V} \times \mathrm{N} \times 0,09}{\mathrm{P}} \times \mathbf{1 0 0}$, Avec $: \mathrm{V}$, le volume ajouté d'hydroxyde de sodium ; $N$, la normalité et $P$, le poids du lait utilisé.

Pour la détermination de la teneur en protéine, la teneur en Azote total a été calculé par la méthode Kjeldhal et multplié par le facteur de conversion de 6,25.

Analyse des paramètres sensoriels: Un test hédonique a été mené pour mesurer le degré d'appréciation des fromages. La dégustation s'est déroulée dans une salle avec des conditions de température et de luminosité identique pour tous les dégustateurs. Au total 23 dégustateurs ont reçu chacun 12 échantillons correspondant aux différents endroits de provenance, une fiche de notation et une bouteille d'eau pour se rincer la bouche entre les différentes dégustations. Des instructions ont été transmises aux dégustateurs sur la façon de remplir la fiche selon leur appréciation en rapport avec la saveur (goût), la texture (aspect) et la couleur. Pour le test hédonique, les paramètres évalués ont étés cotés suivant une échelle de 1 à 9 , ci-après comme décrit par Watts et al. (1991) :

1. Je déteste

2. Je n'aime pas du tout

3. Je n'aime pas

4. Je n'aime pas beaucoup

5. Je suis indifférent

6. J'aime un peu 
7. J'aime modérément

8. J'aime beaucoup
9. J'adore

Calcul du bilan : i

Rendement fromager en $\%)=\frac{\text { Quantité de lait }(\mathrm{kg})}{\text { Quantité de fromage }(\mathrm{kg})} * 100 \quad$ Équation 1

Analyses statistiques: Les données ont été analysées en utilisant les logiciels SPSS 20 et Excel

\section{RÉSULTATS ET DISCUSSION}

Suivi du processus de fabrication du mashanza pendant 24 heures

Température et humidité relative dans l'enceinte de pour déterminer les statistiques de base.

fabrication: Les conditions ambiantes pendant 24 heures dans l'enceinte de fabrication du Mashanza pour les 12 fermes sont reprises au tableau 3 .

Tableau 3 : Température $\left({ }^{\circ} \mathrm{C}\right)$ et humidité relative (\%) dans l'enceinte de fabrication du « Mashanza »

\begin{tabular}{|c|c|c|c|c|c|}
\hline \multirow{3}{*}{ Localisation } & \multirow{2}{*}{ Unité } & \multicolumn{2}{|c|}{ Température $\left.{ }^{\circ} \mathrm{C}\right)$} & \multicolumn{2}{c|}{$\begin{array}{l}\text { Humidité relative (\%) } \\
\text { relative (\%) }\end{array}$} \\
\cline { 3 - 6 } & & Max & Min & Max & Min \\
\hline \multirow{3}{*}{ Kalehe } & Luz & 31 & 19,3 & 72 & 52 \\
\cline { 2 - 6 } & Luk & 30 & 17,6 & 86 & 63 \\
\cline { 2 - 6 } & Mag & 31 & 19,3 & 72 & 52 \\
\hline \multirow{3}{*}{ Kabare } & UCB & 35,9 & 19,1 & 99 & 35 \\
\cline { 2 - 6 } & Comis & 23,9 & 19,1 & 52 & 35 \\
\cline { 2 - 6 } & Miti & 27 & 19 & 99 & 49 \\
\hline \multirow{3}{*}{ Walungu } & Uprelo & 28,5 & 18,4 & 74 & 54 \\
\cline { 2 - 6 } & CAB & 18,8 & 15,1 & 75 & 34 \\
\cline { 2 - 6 } & Cing & 28,5 & 18,4 & 74 & 54 \\
\hline \multirow{3}{*}{ Uvira } & Kond & 34,8 & 26,4 & 59 & 35 \\
\cline { 2 - 6 } & Shuk & 35,9 & 26,3 & 60 & 35 \\
\cline { 2 - 6 } & Zia & 33 & 26,4 & 65 & 35 \\
\hline
\end{tabular}

On observe que les basses températures ont prévalu à Walungu, Kabare et Kalehe et les températures relativement élevées, à Uvira, inversement pour l'humidité relative. Ceci est normal car l'étude a été réalisée durant la saison pluvieuse, mais Uvira se situant en basse altitude, connaît des températures relativement élevées. Selon le Ministère du plan (2005), elles varient entre 20 et $30^{\circ} \mathrm{C}$ dans la plaine de la Ruzizi. Cependant, le type de construction et d'aménagement dans l'enceinte de fabrication peut contribuer à tempérer les conditions ambiantes comme l'absence ou la présence d'ouvertures (portes, fenêtres), les types de toiture, la nature de la cuve de fermentation.

Acidification : $\mathrm{pH}$ et température dans la masse : La figure 1 montre l'évolution de la température du lait pendant le processus de fermentation. En effet, suivant que l'on procède ou non à la pasteurisation du lait avant inoculation et en fonction de la température ambiante dans la salle de fabrication, les valeurs de température peuvent être affectées. On observe une stabilisation de la température jusqu'à la fin du processus (24 heures) entre 12 et $30^{\circ} \mathrm{C}$. Mais les valeurs basses à la fin de la fermentation sont obtenues pour les unités se trouvant à Walungu, précisément à Mulumemunene, entre 2400 et $2500 \mathrm{~m}$ d'altitude (tableau 1). Les fromages frais font intervenir les bactéries lactiques mésophiles, la coagulation se situe entre 25 et $35^{\circ} \mathrm{C}$ (Jamet, 2009) ou 18 à $28^{\circ} \mathrm{C}$ (Mahaut et al., 2000). Ainsi, une température de $40^{\circ} \mathrm{C}$ après inoculation du ferment, indique un lait non tempéré après chauffage. Les températures élevées lors de l'inoculation peuvent perturber les organismes fermentaires. 


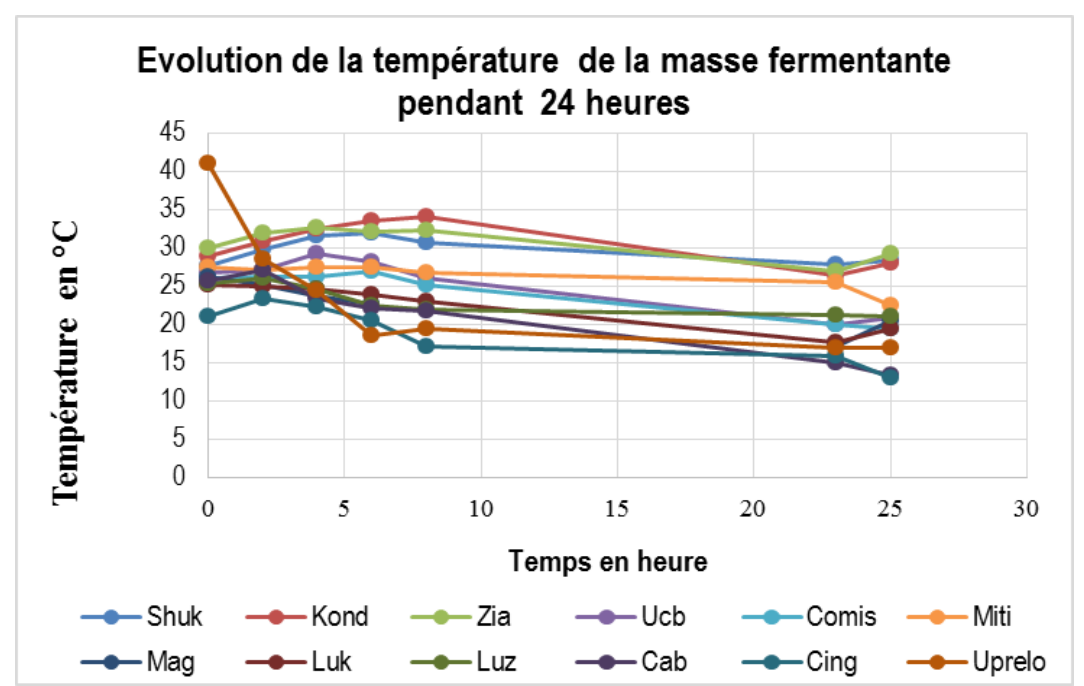

Figure 1: Evolution de la température en fonction du temps durant la fermentation du Mashanza dans les 12 fermes

L'évolution du $\mathrm{pH}$ du lait pendant le processus de fermentation est illustré à la figure 2. Les résultats montrent qu'à la fin de la fermentation, les valeurs de $\mathrm{pH}$ sont comprises entre 3 et 4 . Au cours des 6 heures après inoculation, le $\mathrm{pH}$ chute drastiquement (3,7 à 5$)$, puis diminue à une vitesse lente. La quantité et la qualité de ferment peuvent justifier la courte phase de latence et la vitesse rapide d'acidification au début de la fermentation. Quelques études réalisées sur le Mashanza ont montré des valeurs similaires allant jusqu'au pH 3,5 en fin d'égouttage (Mushayuma, 2011; Cirhuza, 2012). Selon Ramet (1985), Les pâtes fraîches possèdent des valeurs de $\mathrm{pH}$ variant entre 4,3 et 4,5. La croissance des microorganismes peut être influencée par plusieurs facteurs du milieu tels que le $\mathrm{pH}$, la température, la quantité d'eau libre, la concentration en nutriments, la présence des substances antimicrobiennes, ainsi que les interactions entre microorganismes (Laithier et al., 2009). La plupart des aliments fermentés font intervenir les bactéries lactiques (Pilet et al., 2005) qui produisant de l'acide lactique comme produit principal du métabolisme (Dortu \& Thonart (2009) conduisant à une diminution du pH favorable à la conservation des aliments (Labioui et al., 2005). Combiné à l'appauvrissement des sources de carbones fermentescibles, l'abaissement du $\mathrm{pH}$ influence fortement le développement des flores potentiellement pathogènes et ou d'altération, dont l'inhibition sera d'autant plus efficace que l'acidification sera forte et rapide (Jamet, 2009). La croissance bactérienne et l'acidification atteignent leur maxima à la fin de l'égouttage. L'optimisation de la température par ajustement de la température du lait et de l'ambiance pendant l'égouttage permet l'obtention des cinétiques d'évolution optimale (Ramet, 1985). L'acidification participe à la flaveur et à la texture des fromages (Pilet et al., 2005). 


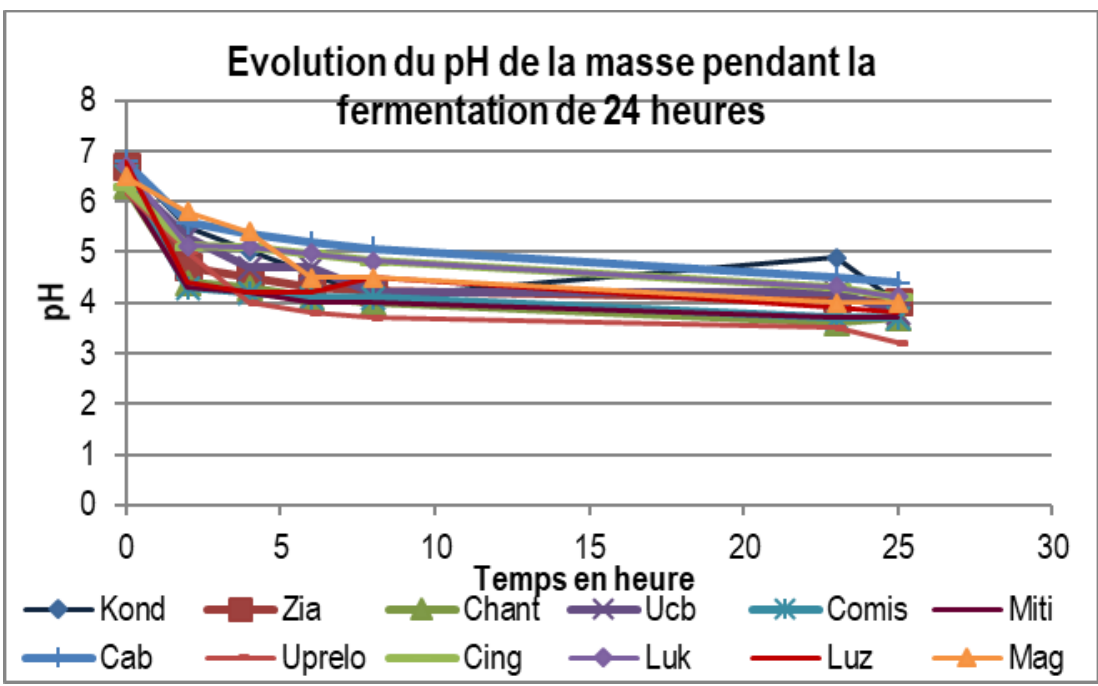

Figure 2 : Evolution du pH durant la fermentation du Mashanza dans 12 fermes pendant $24 \mathrm{~h}$

Comparaison des procédés de fabrication dans les

12 unités: La figure 3 donne le diagramme standard de fabrication de Mashanza utilisé par les 12 unités de fabrication, avec des variantes, comme illustrées sur la figure 4. La figure 4 donne le diagramme standard de fabrication de Mashanza utilisé par les 12 unités de fabrication, avec des variantes.

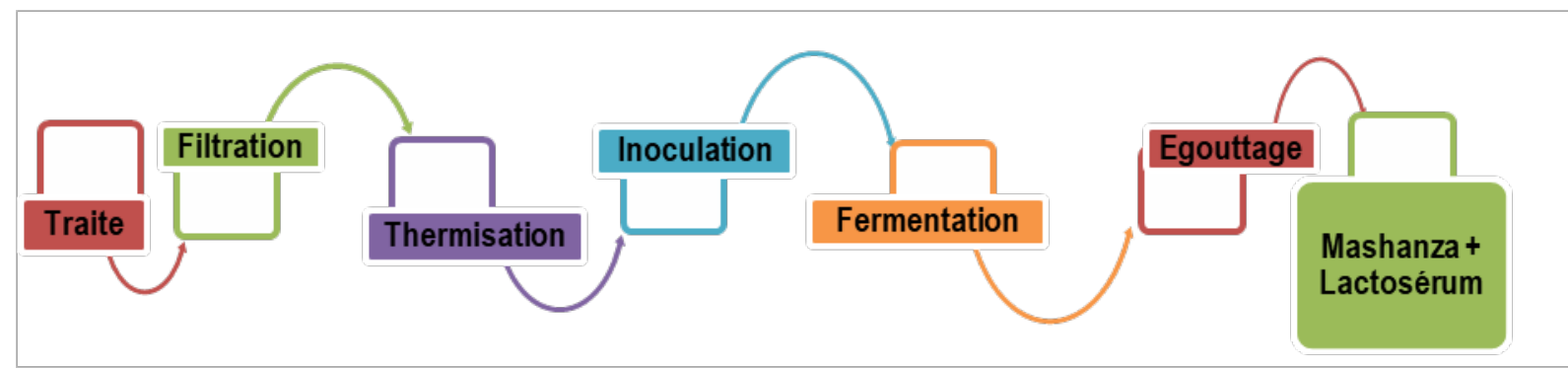

Figure 3: Schéma de fabrication du Mashanza d'après les résultats d'entretiens avec les fabricants

Pendant la traite le lait peut être filtré $(58,3 \%$ de fabricants) ou non. Ensuite, le lait est mélangé au ferment dans une cuve en plastique ou en métal. A Uvira, le lait transformé est acheté dans les fermes. La thermisation est une étape facultative pratiquée dans $50 \%$ des unités de fabrication. Le lait est chauffé à des durées variables, allant de quelques minutes à 20 minutes selon que le chauffage est doux ou poussé, ie jusqu'à la formation d'une mousse ou d'une écume à la surface du lait, le barème n'étant pas contrôlé. Mahaut et cie (2000) recommandent des traitements thermiques modérés $\left(72^{\circ} \mathrm{C}\right.$ pendant 15 à 20 secondes ; $63^{\circ} \mathrm{C}$ pendant 15 à 20 secondes) pour réduire les risques relatives à la perte d'aptitude à la coagulation du lait. L'inoculation se fait en pied de cuve (10 fermes sur 12) ou soit en pied et tête de cuve dans des récipients généralement couverts. On inocule le lait encore tiède pour permettre un bon démarrage de la fermentation. Le ratio lait/ferment de 10:5 est utilisé dans $58,3 \%$ d'unités. D'autres ratios sont utilisés, à savoir : $10: 3,10: 7,10: 8$ et $10: 12$. Les équipements utilisés sont variables avec une prédominance des seaux plastiques avec couvercle de grande dimension et munis des vannes pour l'égouttage. Mais on note aussi l'usage des cruches, des bidons coupés et des calebasses dans quelques fermes. La fermentation dure un jour pour $41,7 \%$ des fabricants. Toutefois, étant donné des problèmes de conservation et d'insuffisance de lait, la durée de fermentation peut s'étendre jusque 7 jours en ajoutant journalièrement du lait dans la cuve de fermentation et en soutirant le lactosérum, éventuellement. La fermentation continue est une solution alternative à ces deux problèmes majeurs. Mais, il reste à étudier les effets d'une telle fermentation sur la qualité et le rendement du Mashanza. Selon Mahaut et al. (2000), la durée de coagulation varie de 
14 à 48 heures pour les pâtes fraîches. L'égouttage est réalisée à l'aide d'une tige en bois (bambou) appelée « murwanzo » (pratiqué par 33,3\% de fabricants), à la louche $(8,3 \%)$, à la main $(25 \%)$, à l'aide d'une faisselle $(16,7 \%)$ et enfin par cavitation $(16,7 \%)$ en utilisant des tanks munis d'une petite ouverture ou d'une vanne dans le bas du récipient comme décrit par Birali ${ }^{2} \&$ cie (2019). Enfin, le Mashanza est conditionné dans des emballages et le lactosérum conservé en attendant une prochaine fabrication du fromage.

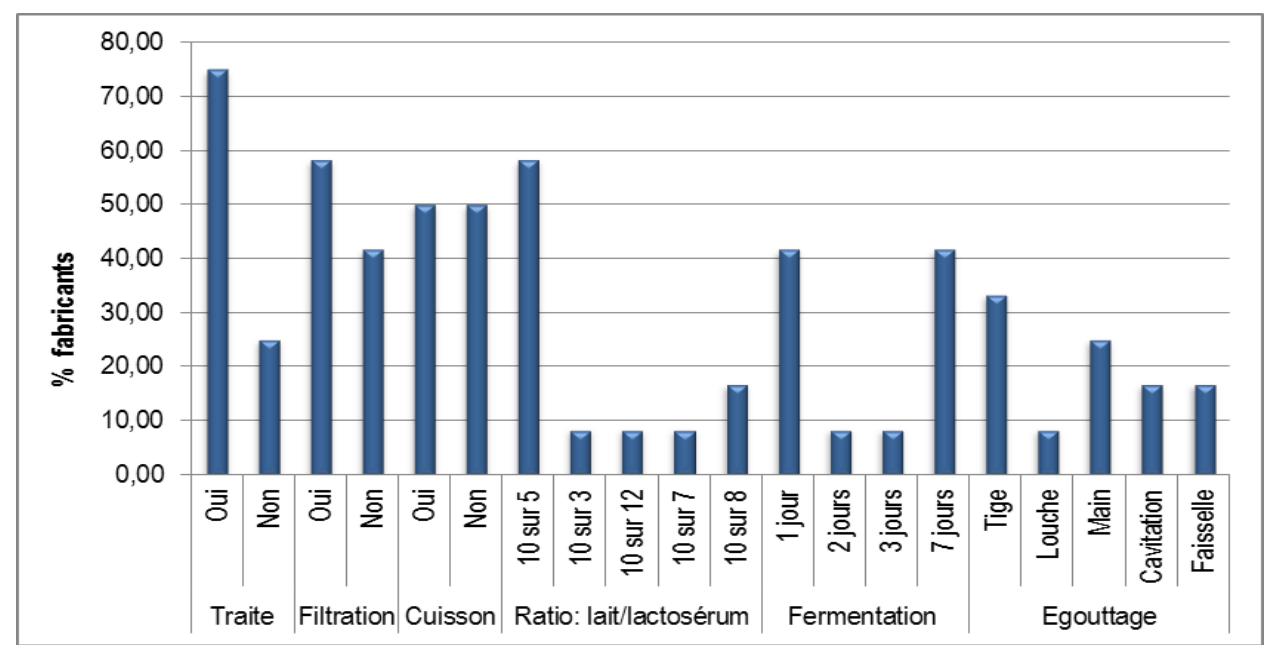

Figure 4 : Modalités de fabrication du Mashanza dans 12 unités de fabrication.

Ainsi, au regard des schémas adoptés par les fabricants, les étapes suivies sont presque les mêmes, les variantes étant la traite, la filtration et la thermisation qui peuvent être facultatives. A ce niveau, on peut suggérer que la filtration soit une étape obligatoire car son rôle dans la prévention de certains dangers physiques (poils, insectes) est avéré. Par contre, le chauffage du lait peut être réalisé lorsque les conditions le permettent afin d'assurer une certaine qualité microbiologique et technologique en tenant compte des barèmes requis comme recommandé par Mahaut \& cie (2000). Cependant pour les fromages fermiers, le traitement thermique peut être omis lorsque le lait est de bonne qualité.

Paramètres quantitatifs: Bilan et rendement technologique. Le tableau 5 donne Paramètres de production du Mashanza dans 9 unités de fabrication.

Tableau 4 : Paramètres de production du Mashanza dans 9 unités de fabrication

\begin{tabular}{|l|c|c|c|c|c|c|c|c|c|}
\hline \multicolumn{1}{|c|}{ Paramètre } & Zia & Shuk & Kond & Uprelo & Cab & Luk & Luz & Comis & Miti \\
\hline $\begin{array}{l}\text { Quantité totale de lait (litre) } \\
\text { transformé en 14 jours }\end{array}$ & 705 & 280 & 105 & 147 & 96,5 & 392 & 268 & 142 & 420 \\
\hline $\begin{array}{l}\text { Quantité totale de lactosérum } \\
\text { (litre) }\end{array}$ & 352 & 140 & 52,5 & 48,3 & 255 & 255 & 343 & 71 & 210 \\
\hline $\begin{array}{l}\text { Quantité lactosérum égoutté } \\
\text { total (litre) }\end{array}$ & 704 & 280 & 105 & 69,0 & 69,0 & 160,5 & 586,9 & 146,3 & 449,4 \\
\hline $\begin{array}{l}\text { Quantité totale de Mashanza } \\
\text { (kg) }\end{array}$ & 312 & 112 & 42 & 23,5 & 19,1 & 79,7 & 61,6 & 28,4 & 84 \\
\hline $\begin{array}{l}\text { Quantité moyenne de } \\
\text { Mashanza/production (kg) }\end{array}$ & 22,3 & 8 & 3 & 1,7 & 1,4 & 5,7 & 4,4 & 2,0 & 6 \\
\hline Rendement fromager (\%) & $\mathbf{4 4 , 2 6}$ & $\mathbf{4 0 , 0 0}$ & $\mathbf{4 0 , 0 0}$ & $\mathbf{1 6 , 0 0}$ & $\mathbf{1 9 , 7 9}$ & $\mathbf{2 0 , 3 3}$ & $\mathbf{2 3 , 0 0}$ & $\mathbf{2 0 , 0 0}$ & $\mathbf{2 0 , 0 0}$ \\
\hline
\end{tabular}

Les résultats relatifs au bilan (input et output) du processus de fabrication du Mashanza montrent qu'à Uvira, l'atelier Zia transforme une grande quantité de lait par rapport aux deux autres au cours de 14 jours. Dans les autres entités, l'atelier de Miti est suivi de Luk, Luz, Comis, Uprelo et Cab. Les quantités de 
lactosérum utilisées dépendent d'une ferme à une autre. Le rendement fromager obtenu après 24 heures de fermentation, ou au-delà est variable, allant de 16 à $44,3 \%$; les valeurs maximales ont été observées à Uvira où l'égouttage se fait à la main, après une fermentation de 24 heures. Ailleurs, les valeurs les plus fréquentes sont autour de $20 \%$. Birali ${ }^{1}$ et al. (2019) ont rapporté de valeurs élevées, dépassant $40 \%$ pour ce type de fromage fabriqué dans les mêmes contrées par plusieurs fermes. Les valeurs moyennes pour les pâtes fraîches sont dans la gamme de $25-35 \mathrm{~kg}$ pour 100 litres de lait (Ramet, 1985). Le rendement fromager dépend essentiellement de la teneur en caséines ou protéines coagulables (Cuvillier, 2005). Toute altération du lait sur le plan microbien ou enzymatique se traduit par une solubilisation des protéines et une dégradation de la matière grasse abaissant le taux de matière sèche dans le fromage. Le chauffage du lait, par sa durée et le choix des températures, influence la quantité de protéines solubles précipitées (Ramet, 1985). Toutefois, les écarts de quantité peuvent être dus à la teneur en eau après l'égouttage et engendrer des écarts de rendement (Goudédranche et al., 2001). Paramètres physico-chimiques : Le tableau 4 donne les résultats des paramètres physico-chimiques.

Tableau 5 : Résultats des paramètres physico-chimiques des échantillons de Mashanza produit dans les 12 unités de fabrication localisées à Kabare, Kalehe, Uvira et Walungu.

\begin{tabular}{|c|c|c|c|c|c|c|c|c|c|}
\hline \multirow{2}{*}{ Structure } & $P$ & $\mathrm{Na}$ & K & MS & Cendres & \multirow{2}{*}{$\mathrm{pH}$} & Protéine & \multirow{2}{*}{$A w$} & Acidité \\
\hline & $m g / g M S$ & $\mathrm{mg} / \mathrm{gMS}$ & $\mathrm{mg} / \mathrm{g} M S$ & $\%$ & $\%$ & & $\%$ & & $\left({ }^{\circ} D\right)$ \\
\hline Kond & $5,5 \pm \quad 0,2$ & $1,8 \pm 1,2$ & $11,6 \pm 1,9$ & $35,5 \pm \quad 0,8$ & $3,3 \pm \quad 0,3$ & 3,5 & $37,2 \pm \quad 0,2$ & 0,79 & $65,9 \pm 1,1$ \\
\hline Shuk & $5,3 \pm \quad 0,5$ & $3,0 \pm 1,7$ & $17,5 \pm 7,0$ & $35,5 \pm \quad 0,1$ & $3,2 \pm \quad 0,2$ & 3,6 & $43,0 \pm \quad 0,2$ & 0,93 & $71,1 \pm 1,0$ \\
\hline Zia & $5,7 \pm 0,5$ & $2,8 \pm \quad 0,9$ & $11,9 \pm 2,1$ & $31,2 \pm \quad 0,6$ & $3,9 \pm \quad 0,4$ & 3,6 & $37,1 \pm \quad 0,2$ & 0,89 & $51,8 \pm \quad 0,1$ \\
\hline Uprelo & $5,5 \pm 1,1$ & $2,8 \pm 1,3$ & $14,6 \pm 1,8$ & $47,1 \pm \quad 0,1$ & $3,6 \pm \quad 0,9$ & 3,5 & $28,3 \pm \quad 0,0$ & 0,92 & $41,5 \pm \quad 0,4$ \\
\hline Luk & $5,7 \pm \quad 0,5$ & $2, \pm 0,0$ & $15,2 \pm \quad 0,4$ & $31,1 \pm 2,2$ & $3,0 \pm \quad 0,1$ & 3,6 & $36,0 \pm \quad 0,2$ & 0,84 & $74,6 \pm \quad 0,6$ \\
\hline Luz & $5,7 \pm 0,1$ & $3,3 \pm 1,1$ & $13,4 \pm 2,7$ & $42,4 \pm 2,5$ & $2,5 \pm \quad 0,1$ & 3,6 & $29,2 \pm \quad 0,2$ & 0,84 & $61,0 \pm 0,2$ \\
\hline Miti & $6,0 \pm 0,1$ & $2,6 \pm \quad 0,6$ & $13,1 \pm 4,7$ & $48,8 \pm \quad 1,8$ & $3,4 \pm \quad 0,2$ & 3,9 & $32,0 \pm \quad 0,2$ & 0,81 & $50,5 \pm \quad 0,2$ \\
\hline UCB & $6,2 \pm 0,2$ & $2,6 \pm \quad 0,2$ & $15,1 \pm 4,0$ & $36,2 \pm \quad 0,5$ & $3,4 \pm \quad 0,2$ & 3,7 & $31,4 \pm \quad 0,0$ & 0,81 & $67,2 \pm \quad 0,1$ \\
\hline Comis & $6,1 \pm 0,1$ & $3,3 \pm \quad 0,9$ & $14,7 \pm \quad 0,4$ & $38,3 \pm 1,6$ & $2,7 \pm \quad 0,1$ & 3,5 & $20,8 \pm \quad 0,0$ & 0,89 & $94,4 \pm \quad 0,5$ \\
\hline Mag & $5,4 \pm 0,1$ & $1, \pm 0,0$ & $5,7 \pm 0,3$ & $38,3 \pm \quad 0,3$ & $3,7 \pm \quad 0,0$ & 3,7 & $39,9 \pm 0,0$ & 0,88 & $59,0 \pm 0,7$ \\
\hline CAB & $4,9 \pm 0,1$ & $0,8 \pm 0,1$ & $5,5 \pm \quad 0,2$ & $36,9 \pm 0,1$ & $3,8 \pm \quad 0,0$ & 3,8 & $38,2 \pm \quad 0,0$ & 0,93 & $100,8 \pm 2,4$ \\
\hline Cing & $5,8 \pm 0,1$ & $1,3 \pm \quad 0,0$ & $6,7 \pm \quad 0,1$ & 34,9 & 3,6 & 3,7 & $21,0 \pm \quad 0,4$ & 0,95 & $68,8 \pm 0,6$ \\
\hline
\end{tabular}

Les valeurs moyennes trouvées sont dans la gamme de 31 à 48,8 \% de matière sèche, les protéines de 20 à $43 \%$, les cendres totaux, de 2,5 à $3,9 \%$ avec les valeurs en $\mathrm{mg} / \mathrm{g}$ de matière sèche variant de 4,9 à 6,2 pour le phosphore, de 0,8 à 3,3 pour le Sodium et de 5,7 à 17,5 pour le Potassium. Le pH varie de 3,5 à 3,9 , l'activité de l'eau (Aw) de 0,79 à 0,95 et enfin, l'acidité lactique, de $41,5^{\circ} \mathrm{D}$ à $100,8^{\circ} \mathrm{D}$. On constate que d'un paramètre à un autre, les valeurs sont variables en fonction, soit des matières premières, soit des procédés utilisés. Ainsi, pour le contenu en matière sèche, les valeurs trouvées sont supérieures à celles préconisées par FAO (1998) comprises entre 20 et $25 \%$. Et pour le $\mathrm{pH}$ et Aw, les valeurs prévues sont respectivement dans la gamme de 4,3 à 4,5 et de 0,980 à 0,995
(Mahaut et al., 2000). Zagabe (2016), dans une étude sur le Mashanza, a trouvé les valeurs de protéine, allant de 4,14 à $10,31 \%$, de 0,14 à $5,4 \%$ de cendres, de 73,5 à $76,5 \%$, de teneur en eau, de 3,11 à 3,5 de $p H$ et de 25,17 à $40,04^{\circ} \mathrm{D}$ d'acidité. Mupole (2016) a trouvé les résultats similaires pour ces différents paramètres. FAO (1998) a montré que le coagulum lactique possède des valeurs comprises entre 65 à $100^{\circ} \mathrm{D}$. Le $\mathrm{pH}$ et l'acidité diffèrent d'une ferme à une autre. Les valeurs trouvées peuvent se justifier par la durée de fermentation variable, la qualité et la quantité de ferment utilisées. Par contre, la matière sèche et l'activité de l'eau peuvent résulter des différents modes d'égouttage et des traitements thermiques. 
Paramètres sensoriels : analyse hédonique

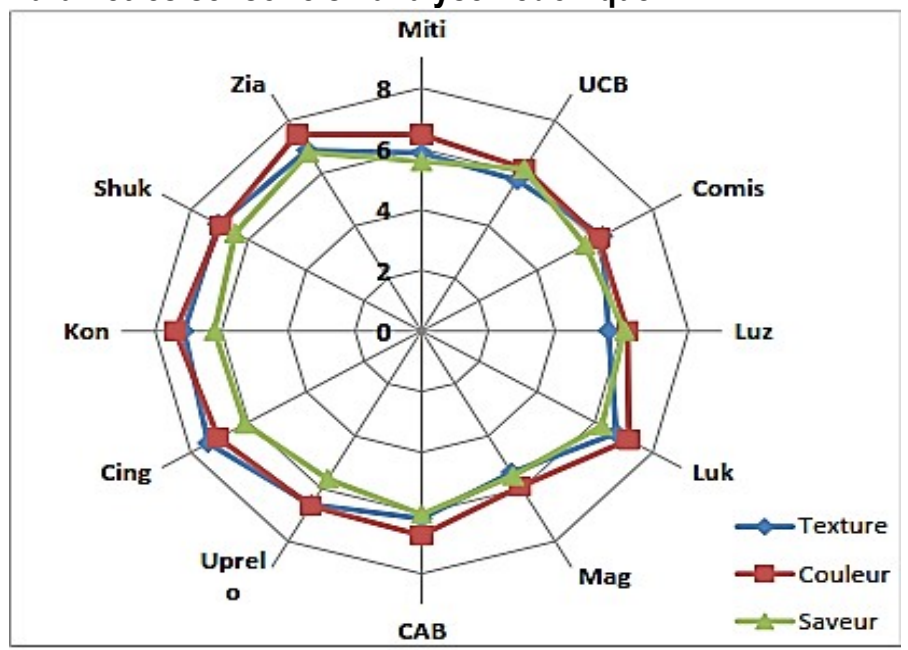

Figure 5 : Barycentres pour la texture, la saveur l'odeur et la couleur du « Mashanza » sur une échelle de 0 à 9

II ressort que tous les fromages ont des moyennes de cotes comprises entre 6 et 7 sur les 3 paramètres. Ceux ayant totalisé des valeurs élevées (7) au niveau de la texture et de la couleur sont ceux produits par Zia, Shu, Kond, Cing, Uprelo. Au niveau de la saveur, le fromage fabriqué par Zia possède un barycentre de 7 , contrairement à tous les autres fromages qui ont une moyenne de 6 . Ce qui peut se justifier par la valeur faible d'acidité lactique (autour de $51,8^{\circ} \mathrm{D}$ ). Mais dans l'ensemble tous les fromages ont été bien appréciés. Dans une étude réalisée par Birali ${ }^{2} \&$ cie (2019) sur le Mashanza, la majorité des fromages produits par les fabricants à Kabare, Kalehe, Walungu et Uvira ont été classés par eux dans la catégorie à consistance ferme et intermédiaire, de coloration blanche, blanc-crème ou

\section{CONCLUSION ET PERSPECTIVES}

L'analyse des résultats a montré que les procédés utilisés par les 12 unités de fabrication varient en fonction de la source d'approvisionnement du lait, du traitement thermique, de la quantité de lactosérum utilisée comme ferment, de la durée de fermentation, des récipients utilisés ainsi que du mode d'égouttage. Concernant l'évaluation sensorielle des fromages, en général, tous ont été bien appréciés. Les résultats obtenus permettront de servir de base pour la recherche de la norme du Mashanza dont la qualité RÉMERCIEMENTS

Les auteurs tiennent à remercier le VLIR pour son soutien financier, l'Ingénieur Bienvenu Rukiranuka et jaunâtre et de saveur variant du neutre, douce, aigre, sucrée, intermédiaire entre sucrée et aigre ou indescriptible pour certains et l'odeur variait de normale, à l'odeur de fermenté, ou de lait. La consistance peut dépendre du contenu élevé en eau combiné au faible degré de minéralisation pouvant entraîner un manque de tenue et de cohésion du fromage (FAO, 1998). Ramet (1985) montre que la friabilité du fromage dépend de différents facteurs technologiques. L'incidence de l'égouttage sur la texture des fromages a été rapportée par Laithier et al. (2009). Depledt et Sauvageot (2002) justifient les seuils de perception variables comme résultant des différences interindividuelles de sensibilité ou d'acceptabilité entre sujets.

n'était pas bien maîtrisée jusque-là. Ils peuvent servir à orienter le choix des procédés pouvant s'adapter au niveau d'investissement des producteurs en prenant en compte les facteurs influençant la qualité du produit final. Ainsi, l'effet de la durée de la fermentation et des modes d'égouttage sur les caractéristiques nutritionnelles, sensorielles et microbiologiques et sur le rendement fromager mérite d'être approfondi dans des études ultérieures.

Monsieur Jean-Charles Cirhuza pour leur collaboration. 


\section{RÉFÉRENCES BIBLIOGRAPHIQUES}

Birali. M., Sumbu, Z. E., \& Walangululu, M.J., 2019. « Note de recherche : Fabrication du fromage frais «Mashanza» produit à Bwegera (Uvira), Kabare, Kalehe et Walungu ", Revue des sciences agronomiques et d'ingénierie biologique appliquée du Kivu, Harmattan, $p$.

Birali, M., Sumbu, Z. E. et Walangululu, M. J., 2019. Diagnostic des procédés de fabrication du fromage blanc traditionnel «mashanza» dans les zones productrices du Sud-Kivu, J. Appl. Biosci., 138: 14017 - 14028pp.

Cirhuza, J.-C., 2012. Etude de diagnostic des procédés traditionnels de fabrication de «Mashanza» et leur impact sur la contamination microbienne et l'évaluation de la qualité physico-chimique et organoleptique des produits obtenus: cas de «Mashanza» fabriqué à Kalehe. Mémoire: Université Catholique de Bukavu (Bukavu), inédit, 74pp.

Cuvillier, D., 2005. Améliorer le rendement fromager. http://cfbourgogne.free.fr//MG/pdf/fiche_ameliorer _rendement.pdf, consulté le 01/5/2019, 6pp.

Depledt, F. et Sauvageot, F., 2002. Évaluation sensorielle des produits alimentaires. Technique de l'ingénieur, traité Agroalimentaire, F4000 v1, $7 p p$.

Diop, P.E.H., 1996. Reproduction et production laitière. AUPELF-UREF. Tunis. $316 \mathrm{pp}$ : 63-75pp.

Dortu, C., \& Thonart, P., 2009. Les bactériocines des bactéries lactiques : caractéristiques et intérêts pour la bioconservation des produits alimentaires. Biotechnol. Agron. Soc. Environ., 1(13), 143$154 \mathrm{pp}$.

FAO, 1998. Le lait et les produits laitiers dans la nutrition humaine. http://www.fao.org/docrep It4280f/T4280FOf.htm\#Chapitre\%206\%20Fromag es, 255pp. consulté le 8.04.2019.

Goudédranche, H., Camier-Caudron -B., Gassi, J-Y \& Schuck, P. 2001. Procédé de transformation fromagère (partie 1). Technique de l'ingénieur, traité Agroalimentaire, $\mathrm{F} 6305,11 \mathrm{pp}$.

Brulé, G. 2008. Science des aliments. Biochimie Microbiologie - Procédés - Produits. Vol. 2: Technologie des produits alimentaires, Technique \& Documentation, Paris. 456pp.

Jamet, E., 2009. Les bactéries lactiques: une composante de l'écosystème microbien des fromages. In: Drider, D.J \& Prévost, H., (Eds.) Bactéries lactiques, Economica, Paris, 319343pp.
Labioui, H., Elmoualdi, L., El Yachioui, M., \& Ouhssine, M., 2005. Sélection de souches de bactéries lactiques antibactériennes. Bull. Soc. Pharm. Bordeaux, (144), 237-250pp.

Laithier, C., Chatelin, Y.M. , Doutart, E., Barrucand, P., Duchesne, C., Morge, S., Barral, J, Cuvillier, D., Minard, L., Leroux, V., 2009. Evaluation et maîtrise de la texture des fromages frais de chèvre à coagulation lactique, Renc. Rech. Ruminants, (16) 143-146pp.

Mahaut, M., Jeantet, R., \& Brulé, G., 2000. Initiation à la technologie fromagère. Paris: Technique et Documentation. 194pp.

Ministère du Plan, 2005. Monographie de la province du Sud-Kivu, PNUD-UNOPS, 179pp.

Mupole, C., 2016. Etude de l'effet de la thermisation et des modes d'égouttage sur la qualité et la durée de conservation du Mashanza, Mémoire: Université Catholique de Bukavu (Bukavu)», inédit, 45pp.

Mushayuma, C., 2011. Effet de la pasteurisation sur les paramètres organoleptiques du «Mashanza». «Travail de fin de cycle: Université Catholique de Bukavu (Bukavu) », inédit, 38pp.

Pilet, M.-F., Magras, C. \& Federighi, M., 2005. Bactéris lactiques. In: Bactériologie alimentaire. Compendium d'hygiène des aliments. Economica, Paris, 219-240pp.

Ramet, J.P., 1985. La fromagerie et les variétés de fromages du bassin Méditerranéen. Etude FAO. Production et santé animale (48), http://www.fao.org/docrep/004/X6551F/X6551F00. HTM, (12/12/2018), 187pp.

Vivegnis, J., Dubois, C., Nicolay, L., Mairy, F., Jacob, C., Piraux, E., El Lioui, M., Decalonne, J., 1998. Qualité microbiologique des fromages artisanaux fabriqués au lait cru en Région wallonne, Biotechnol. Agron. Soc. Environ. 2 (4), 248255pp.

Watts, BM, Ylimaki, GL, Jeffery, LE \& Elias, LG, 1991. Méthodes de base pour l'évaluation sensorielle des aliments. Centre de recherches pour le développement international (CRDI), Ottawa, 143pp.

Zagabe, D., 2016. Contribution à l'étude d'amélioration de la qualité du fromage frais Mashanza produit à la ferme de l'U.C.B à Kavumu, Mémoire: Université Catholique de Bukavu (Bukavu), inédit, 76pp. 